\title{
STIEFEL-WHITNEY HOMOLOGY CLASSES OF QUASI-REGULAR CELL COMPLEXES
}

\author{
RICHARD GOLDSTEIN AND EDWARD C. TURNER
}

\begin{abstract}
A quasi-regular cell complex is defined and shown to have a reasonable barycentric subdivision. In this setting, Whitney's theorem that the $k$-skeleton of the barycentric subdivision of a triangulated $n$-manifold is dual to the $(n-k)$ th Stiefel-Whitney cohomology class is proven, and applied to projective spaces, lens spaces and surfaces.
\end{abstract}

1. $Q R$ complexes. A (finite) cell structure on a space $X$ is defined (see, e.g., [Brown, p. 124]) to be a (finite) family of maps $\left\{\sigma: E^{n_{0}} \rightarrow X\right\}$ called cells so that

(i) $X=\cup_{\sigma}\left\{\sigma\left(\operatorname{int}\left(E^{n_{o}}\right)\right)\right\}$.

(ii) $\sigma \mid \operatorname{int}\left(E^{n_{0}}\right)$ is a homeomorphism onto its image.

(iii) $\sigma\left(\partial E^{n_{\mathrm{a}}}\right) \subset \cup_{n_{\mu}<n_{o}}\left(\mu\left(E^{n_{\mu}}\right)\right)$.

(We will deal with finite complexes throughout for simplicity, although everything holds in the locally finite context.) The cell structure will be called quasi-regular $(Q R)$ if the following condition holds: for each cell $\sigma: E^{n_{0}} \rightarrow X$, there is a cell structure (necessarily unique) on $\partial E^{n_{0}}$ so that for each cell $\omega$ in $\partial E^{n_{o}}, \sigma \circ \omega$ is a cell in $X$; note that this boundary structure must also be $Q R$. Such a structure will be called a $Q R$ complex and $\bar{X}$ will denote the space $X$ with this additional structure. The most familiar example of a $Q R$ structure is the usual cell structure on $\mathbf{R} P^{n}$, denoted $\overline{\mathbf{R} P}^{n}$, with one cell in each dimension. Also, any regular cell complex is $Q R$.

The product of two $Q R$ structures is defined as usual by taking for cells in the product $X \times Y$, products $\left\{\sigma \times \tau: E^{n_{\sigma}} \times E^{n_{+}} \rightarrow X \times Y\right\}$ of cells $\sigma$ and $\tau$ in $X$ and $Y$ respectively, a structure which is easily checked to be $Q R$. Barycentric subdivision can be defined inductively in analogy with the simplicial case as follows: for each $n_{\sigma}$-cell $\sigma$, cone over the cells in the subdivision of the associated $Q R$ structure on $\partial E^{n_{\mathrm{o}}}$ and consider the set of cells so obtained: a $Q R$ complex with only zero cells is its own subdivision. It is not hard to see that the subdivision of a $Q R$ complex $\bar{X}$ is a $Q R$ complex, denoted $\bar{X}^{\prime}$, and is in fact a pseudo-triangulation in the sense of [Hilton and Wiley], so that a second subdivision yields a triangulation. Incidence numbers can also be defined for a $Q R$ complex: if $\sigma$ and $\tau$ are two cells in $X$, then $[\tau, \sigma]$ is the number of cells $\omega$ in the $Q R$ structure on $\partial E^{n_{4}}$ corresponding to $\tau$ so that $\tau \circ \omega=\sigma$. In the barycentric subdivision, the origins of the original

Received by the editors September 23, 1975 and, in revised form, May 17, 1976.

AMS (MOS) subject classifications (1970). Primary 57C99; Secondary 57D20.

Key words and phrases. Euler (mod 2) space, Stiefel-Whitney homology class. 
cells are the vertices of the pseudo-triangulation and $[\tau, \sigma]$ is the number of 1-cells spanned by the origins of $\tau$ and $\sigma($ if $\operatorname{dim}(\tau)>\operatorname{dim}(\sigma))$.

DeFinition.

$$
\begin{aligned}
& X_{0}=\cup\left\{\sigma\left(\operatorname{int}\left(E^{n_{o}}\right)\right) \mid \sum_{\tau}[\tau, \sigma] \equiv 0(\bmod 2)\right\}, \\
& X_{1}=\cup\left\{\sigma\left(\operatorname{int}\left(E^{n_{o}}\right)\right) \mid \sum_{\tau}[\tau, \sigma] \equiv 1(\bmod 2)\right\} .
\end{aligned}
$$

Recall that $\Sigma \operatorname{rank}\left(H\left(X, X-x ; \mathbf{Z}_{2}\right)\right)$ is the local Euler number of $X$ at $x$.

THEOREM 1.

$$
\begin{aligned}
& X_{0}=\{x \mid \text { local Euler number of } X \text { at } x \equiv 1(\bmod 2)\} \\
& X_{1}=\{x \mid \text { local Euler number of } X \text { at } x \equiv 0(\bmod 2)\}
\end{aligned}
$$

The decomposition $X=X_{0} \cup X_{1}$ thus depends only on $X$ and not on the $Q R$ structure.

Proof. A straightforward proof, left to the reader, shows that if $\bar{X}$ is a triangulation, then $\Sigma_{\tau}[\tau, \sigma]$ and $1+\Sigma \operatorname{rank}\left(H^{*}\left(X, X-x ; \mathbf{Z}_{2}\right)\right)$ are both equal to the Euler characteristic of the link of $x$ in $\bar{X}$, mod 2. The following claim will then establish the general case, since two barycentric subdivisions of a $Q R$ structure yield a triangulation.

Claim. The decomposition $X=X_{0} \cup X_{1}$ is invariant under barycentric subdivision.

It is now necessary to introduce some notation for barycentric subdivision that is a direct generalization of the usual for a simplicial complex. With each $k$-cell $\mu$ in $\bar{X}$ we associate a sequence of cells in $\bar{X}$ as follows: if $k=0$, then $\sigma_{0}$ is the unique cell whose interior contains $\mu$ : if $k>0$, then there is a unique $n$-cell $\sigma_{k}$ so that $\mu$ is the image under $\sigma_{k}$ of the cone over some cell $\omega$ in $\partial E^{n}-$ if the $(k-1)$-cell $\sigma_{k} \circ \omega$ is associated with $\left(\sigma_{0}, \ldots, \sigma_{k-1}\right)$, then $\mu$ is associated with $\left(\sigma_{0}, \ldots, \sigma_{k-1}, \sigma_{k}\right)$. Furthermore, if the lifts $\omega_{i}$ of $\sigma_{i-1}$ to $\sigma_{i}$ are specified, the representation is unique-each $k$-cell $\mu$ in $\bar{X}^{\prime}$ corresponds to a sequence $\left\langle\sigma_{0}^{\omega_{1}} \sigma_{1}^{\omega_{2}} \ldots \sigma_{k-1}^{\omega_{k}} \sigma_{k}\right\rangle$. The subcells of $\mu$ as above are those obtained by eliminating various $\sigma_{i}$ 's and composing the corresponding lifts; e.g.

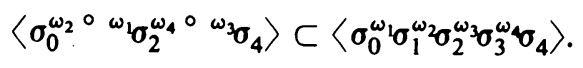

Geometrically, the cell $\sigma_{k}$ is the union of all cells $\mu$ with representatives as above. We need to show, then, that $\Sigma_{\tau}\left[\tau, \sigma_{k}\right]$ in the original structure is equivalent $\bmod 2$ to $\Sigma_{\eta}[\eta, \mu]$ in the subdivision.

The proof of the claim is by induction on $\operatorname{dim}(X)$. \{We wish to thank the referee for pointing out an error in our original proof. $\}$ The case $n=0$ is trivial.

STEP 1 . The number of $\xi>\mu$ obtained by replacting $\sigma_{k}$ with $\sigma_{k}^{\alpha} \tau$ is equivalent $\bmod 2$ to $\Sigma\left[\tau, \sigma_{k}\right]$.

This is clear from the definition. 
STEP 2. The number of $\eta>\mu$ obtained by replacing $\sigma_{0}$ with $\tau^{\alpha} \sigma_{0}$ is even.

The number of such $\eta$ is the number of cells in the structure on $\partial E^{\operatorname{dim}\left(\sigma_{i}\right)}$ which is $\chi\left(\partial E^{\operatorname{dim}\left(\sigma_{i}\right)}\right) \bmod 2$ and thus even.

STEP 3. The number of $\eta>\mu$ obtained by replacing $\sigma_{i-1}^{\omega_{i}} \sigma_{i}$ with $\alpha_{i-1}^{\alpha} \tau^{\beta} \sigma_{i}$ is even.

We are counting the number of ways of choosing the dotted maps below to make the diagram commute.

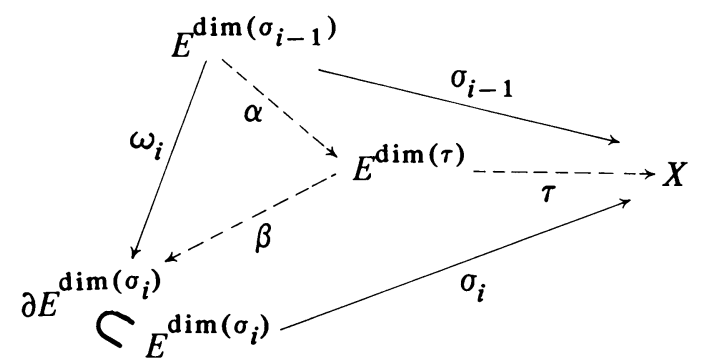

This is the same as choosing $\alpha$ and $\beta$, of which there are $\Sigma\left[\beta, \omega_{i}\right]$ choices (the sum taken in $\left.\partial E^{\operatorname{dim}\left(\sigma_{i}\right)}\right)$. By induction, $\left(\partial E^{\operatorname{dim}\left(\sigma_{i}\right)}\right)_{0}=\partial E^{\operatorname{dim}\left(\sigma_{i}\right)}$ so this sum is even.

STEP 4. The number of $\eta \supset \mu$ is equivalent $\bmod 2$ to $\Sigma\left[\tau, \sigma_{k}\right]$.

To choose $\eta$ requires 0 or 1 choice as in Step 1 and some number of choices as in Steps 2 and 3, making at least one choice. This completes the proof of the claim and the theorem.

Definition. Let $C_{p}(X)$ be the $p$-chain which is the sum of the $p$-cells of the barycentric subdivision $\bar{X}^{\prime}, \bar{X}$ is said to be an Euler $(\bmod 2)$ space if $C_{p}(\bar{X})$ is a $p$-cycle for each $p$, in which case we define $\omega_{p}(\bar{X})=\left[C_{p}(\bar{X})\right] \in H_{p}\left(X ; \mathbf{Z}_{2}\right)$.

ThEOREM 2. $\partial\left(C_{k+1}(\bar{X})\right)=\Sigma\left\{\tau \mid \tau\right.$ is a $k$-cell in $\bar{X}^{\prime}$ whose interior lies in $\left.X_{1}\right\}$.

Corollary 1. $\bar{X}$ is Euier (mod 2) if and only if $X=X_{0}$. The property of being Euler $(\bmod 2)$ is therefore a topological property.

Corollary 2. If $X$ is an Euler $(\bmod 2)$ space, then $\omega_{k}(\bar{X})=\omega_{k}\left(\bar{X}^{\prime}\right)$ in $H_{k}\left(X ; \mathbf{Z}_{2}\right)$.

If $X$ is a manifold, a $Q R$ structure is said to be smooth if its second barycentric subdivision is a smooth triangulation.

COROllary 3. If $\bar{X}$ is a smooth $Q R$ structure on a smooth manifold, then $\omega_{k}(\bar{X})$ is Poincaré dual to the $(n-k)$ th Stiefel-Whitney cohomology class.

Proof of THEOREM. Let $\mu$ be a $k$-cell in $\bar{X}^{\prime}$ represented by $\left\langle\sigma_{0}^{\omega_{1}} \sigma_{1} \ldots{ }^{\omega_{k}} \sigma_{k}\right\rangle$. We must show that the number of $(k+1)$-cells $\eta$ of which $\mu$ is part of the boundary is congruent $\bmod 2$ to $\Sigma_{\xi}[\xi, \mu]=\Sigma_{\tau}\left[\tau, \sigma_{k}\right]$ : i.e., $\mu$ appears in the boundary of $C_{k+1}(\bar{X})$ if and only if $\mu \subset \sigma_{k} \subset X_{1}$. To choose such an $\eta$ is to 
make exactly one choice as in steps 1-3 of Theorem 1. Of these, there are exactly $\Sigma_{\tau}\left[\tau, \sigma_{k}\right] \bmod 2$, proving the theorem.

Proof of Corollary 1 . The $C_{p}(\bar{X})$ are all cycles iff $X_{1}=\varnothing$.

Proof of Corollary 2. Consider the $Q R$ structure on $X \times I$, denoted $\widetilde{X \times I}$, obtained by taking the product of the given structure on $X$ with the obvious one on $I$, and then subdividing $X \times\{1\}$. It is easy to check that if $X$ is $E(2)$, then $(X \times I)_{1}=X \times\{0\} \cup X \times\{1\}$ so that $\partial\left(C_{k+1}(\widetilde{X \times I})\right)=$ $\omega_{k}(X) \times\{0\} \cup \omega_{k}\left(\overline{X^{\prime}}\right) \times\{1\}$. Thus, these two cycles are homologous. (Note. This result appears to be well known but we have not seen it written down. It is probably due to Toledo or Sullivan.)

Proof of Corollary 3. This is well known for triangulations [Halperin and Toledo], and by Corollary 2 , the classes are invariant under subdivision.

2. Applications. In this section, we apply the above ideas to projective spaces, lens spaces and surfaces.

Proposition 1.

$$
\omega_{k}\left(\overline{\mathbf{R} P}^{n}\right)=\left(\begin{array}{c}
n+1 \\
n-k
\end{array}\right) Z_{k} \quad(\bmod 2)
$$

where $Z_{k}$ is the generator of $H_{k}\left(\mathbf{R} P^{n} ; \mathbf{Z}_{2}\right), 0 \leqslant k \leqslant n$.

Proof. $\overline{\mathbf{R}}^{n}$ denotes $\mathbf{R} P^{n}$ with the familiar cell structure with one cell $\sigma_{i}$ in each dimension $i, 0 \leqslant i \leqslant n .\left[\sigma_{i}, \sigma_{i}\right]=2$ for each $i>j$ and we denote the two lifts of $\sigma_{j}$ to $\sigma_{i}$ by 0 and $1-0$ denotes the lift to the upper hemisphere and 1 the composition of 0 with the antipodal map-so that composition of lifts acts like mod 2 addition. The diagram below shows how this scheme works in $\mathbf{R} P^{2}$.

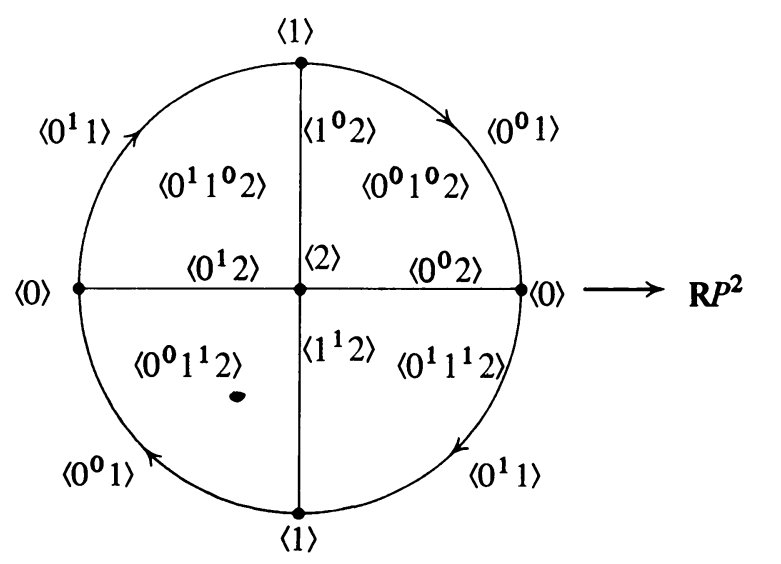

For each sequence $i_{0}<\cdots<i_{k}$ of integers from 0 to $n,\left\langle i_{0} i_{1} \ldots i_{k}\right\rangle$ will denote the chain composed of all $k$-cells in $\overline{\mathbf{R}}^{n}$ whose representations, ignoring lifts, are $\left\langle\sigma_{i_{0}} \sigma_{i_{1}} \ldots \sigma_{i_{k}}\right\rangle$ : the same convention will hold if some, but 
not all, lifts are specified. The fact that $\left[\sigma_{i}, \sigma_{j}\right]$ is always even implies that $\left\langle i_{0} i_{1} \ldots i_{k}\right\rangle$ is a mod 2 cycle. Now $\langle 01 \ldots k\rangle$ clearly represents $Z_{k}$, since, geometrically, it is the standard copy of $\mathbf{R} P^{k}$ in $\mathbf{R} P^{n}$. Furthermore, any other chain $\left\langle i_{0} i_{1} \ldots i_{k}\right\rangle$ is homologous to $\langle 01 \ldots k\rangle$ since their mod 2 difference is the boundary of

$$
\left\langle 0{ }^{0} i_{0} i_{1} \ldots i_{k}\right\rangle+\left\langle 01^{0} i_{1} i_{2} \ldots i_{k}\right\rangle+\left\langle 012^{0} i_{2} \ldots i_{k}\right\rangle+\ldots+\left\langle 012 \ldots k^{0} i_{k}\right\rangle .
$$

(If there is a repeat in any of these terms, e.g., $i_{0}=0$, that term should be ignored.) Since the sum of all the $\left\langle i_{0} \ldots i_{k}\right\rangle$ is the $k$-skeleton of $\overline{\mathbf{R} P}^{n \prime}$ and there are $\left(\begin{array}{c}n+1 \\ k+1\end{array}\right)$ of them,

$$
\omega_{k}\left(\overline{\mathbf{R} P}^{n}\right)=\left(\begin{array}{l}
n+1 \\
k+1
\end{array}\right) Z_{k}=\left(\begin{array}{l}
n+1 \\
n-k
\end{array}\right) Z_{k} .
$$

Now consider a generalized lens space $L=L\left(p ; q_{1}, \ldots, q_{n}\right)$ of dimension $m=2 n-1$. The CW structure described in [Cohen, p. 90] is a $Q R$ structure, denoted $\bar{L}$. Since $L$ has no interesting $\mathbf{Z}_{2}$ homology if $p$ is odd, we assume $p$ is even, in which case $H_{k}\left(L, \mathbf{Z}_{2}\right) \cup \mathbf{Z}_{2}$ for $0 \leqslant k \leqslant m$ with generator $\mathbf{Z}_{k}$.

Proposition 2. $\omega_{k}(\bar{L})=\left(\begin{array}{c}m+1 \\ m-k\end{array}\right) Z_{k}, 0 \leqslant k \leqslant m$.

Proof. The proof generalizes that of Proposition 1. The CW structure has one cell $\sigma_{i}$ in each dimension $0 \leqslant i \leqslant m$ and $\left[\sigma_{i}, \sigma_{j}\right]=p(i>j)$ except for $\left[\sigma_{2 j+1}, \sigma_{2 j}\right]=2$. The various lifts can be denoted by $0, \ldots, p-1$, with the proviso that in the case $\sigma_{2 j} \subset \sigma_{2 j+1}$, only 0 and 1 are allowed, and composition of lifts acts like $\bmod p$ addition. Again $\left\langle i_{0} \ldots i_{k}\right\rangle$ will denote the chain composed of all cells represented, ignoring lifts, by $\left\langle\sigma_{i_{0}} \cdots \sigma_{i_{k}}\right\rangle$-this is a cycle because $\left[\sigma_{i}, \sigma_{j}\right]$ is always even. $\langle 01 \ldots k\rangle$ clearly represents $Z_{k}$ since geometrically, it is the $k$-skeleton of $\bar{L}$. But any such chain is homologous to $\langle 01 \ldots k\rangle$ since $\bmod 2$,

$$
\begin{aligned}
\left\langle i_{0} i_{1} \ldots i_{k}\right\rangle & -\langle 01 \ldots k\rangle \\
& =\partial\left[\left\langle 0^{0} i_{0} i_{1} \ldots i_{k}\right\rangle+\left\langle 01^{0} i_{1} \ldots i_{k}\right\rangle+\cdots+\left\langle 01 \ldots k^{0} i_{k}\right\rangle\right] .
\end{aligned}
$$

(Same convention as in Proposition 1 as to repeated entries.) Therefore, $\omega_{k}(\bar{L})=\left(\begin{array}{c}m+1 \\ m-k\end{array}\right) Z_{k}$.

If $M$ is a connected, compact 2-manifold, then there is a "standard" $Q R$ structure, denoted $\bar{M}$, that corresponds to identifying line segments on the boundary of a 2-disc. The calculations of $\omega_{0}(\bar{M})$ and $\omega_{2}(\bar{M})$ are straightforward and left to the reader. $\omega_{1}(\bar{M})$ can be described as follows: let $Z$ be any class in $H_{1}\left(M, Z_{2}\right)$ represented by an embedding of $S^{1}$ (generators can be so chosen)-then $C_{1}(\bar{M})$ has mod 2 intersection number with $Z$ (generically) iff the embedding has nontrivial normal bundle. On the $m$-handled torus, $\omega_{1}\left(m T^{2}\right)=0$ and the shaded 2-chain below has $C_{1}\left(m T^{2}\right)$ as its boundary. 


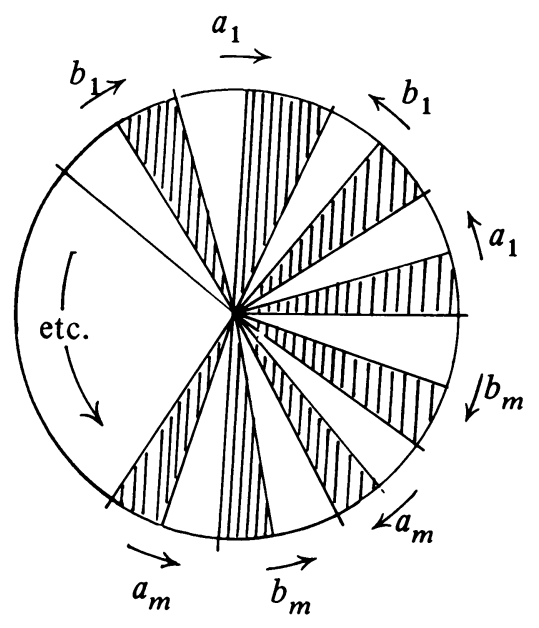

For a multiple projective plane $m P^{2}$, the shaded 2-chain below is an homology between $C_{1}\left(m P^{2}\right)$ and the dotted 1-chain, which clearly has the property described above.

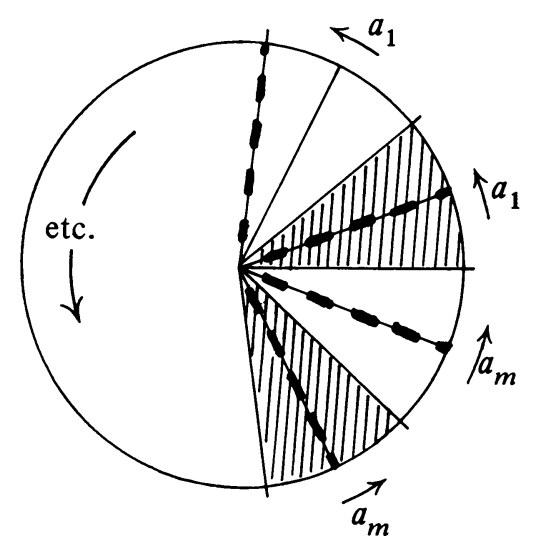

The remaining case of $S^{2}$ is left to the reader.

\section{REFERENCES}

Ronald Brown, Elements of modern topology, McGraw-Hill, New York, 1968. MR 37 \#3563.

M. M. Cohen, $A$ course in simple homotopy theory, Springer-Verlag, Berlin and New York, 1973. MR 50 \# 14762.

S. Halperin and D. Toledo, Stiefel-Whitney homology classes, Ann. of Math. (2) 96 (1972), 511-525. MR 47 \# 1072.

P. J. Hilton and S. Wiley, Homology theory, Cambridge Univ. Press, New York, 1960. MR 22 \#5963.

Department of Mathematics, State University of New York at Albany, Albany, New YORK 12222 